\title{
Downlink Resource Allocation for Multi-user MIMO-OFDMA Systems: The Kalai-Smorodinsky Bargaining Approach
}

\author{
Jie Chen and A. Lee Swindlehurst \\ Department of Electrical Engineering and Computer Science \\ University of California, Irvine \\ Irvine, CA 92697 USA \\ Email: \{jie.chen, swindle\}@uci.edu
}

\begin{abstract}
Multi-user MIMO-OFDMA is regarded as an important technology for increasing the flexibility and efficiency of wireless communication systems. A well-behaved resource allocation strategy is crucial for the performance of such systems. In this paper, we study the allocation problem from a cooperative game theory perspective and present two algorithms to find the Kalai-Smorodinsky bargaining solution to the problem. Simulation results show that the algorithms can achieve a reasonable tradeoff between fairness and efficiency.
\end{abstract}

\section{INTRODUCTION}

As a promising technology to significantly increase the spectral efficiency of wireless systems, multiple-input and multiple-output (MIMO) techniques have drawn much attention over the past two decades. The work of [1], [2] and the references therein have studied the information-theoretic aspects of MIMO systems and showed the capacity region for broadcast (BC) and multiple-access (MAC) channels. Orthogonal frequency division multiple access (OFDMA) is another technique which has attracted substantial research interest. Almost all newly developed wireless systems, such as 3GPP LTE, LTE-Advanced and IEEE $802.16 \mathrm{~m}$, base their air interfaces on MIMO-OFDMA.

MIMO-OFDMA systems can be deployed in two flavors: single-user and multi-user. For communications without feedback or with limited feedback, it is reasonable to adopt a single-user MIMO strategy. For the case that the channel state information (CSI) is known at the transmit side, multi-user MIMO approaches are required if one wishes to exploit the full potential of the diversity offered by MIMO channels. Most proposed capacity-achieving schemes are based on dirty paper coding (DPC) [3], which is non-linear and computationally prohibitive. In fact only linear precoding is considered for modern cellular systems like LTE.

For a multi-user MIMO-OFDMA system, a reasonable allocation of resources, such as power, subcarriers and spatial channels, is crucial to system performance. Numerous papers have focused on maximizing the sum rate of the system [4] [5], while others [6] [7] also considered fairness among the users.

This work was supported by the U.S. Army Research Office under the Multi-University Research Initiative (MURI) grant W91-1NF-04-1-0224.
Recently, researchers have begun to interpret wireless communication problems from a game theory perspective, which provides new mechanisms for solving resource allocation problems. As a branch of cooperative game theory, bargaining theory has been applied to wireless networks [8] [9] in order to attain a useful tradeoff between overall system efficiency and user fairness. In this paper, we focus on the Kalai-Smorodinsky bargaining solution (KSBS) [10] to the problem of downlink resource allocation for multi-user MIMO-OFDMA systems.

\section{SySTEM MODEL}

We consider a system model for the downlink channel with $n_{T}$ transmit antennas and $K$ users, each equipped with $n_{R}^{(k)}$ receive antennas. We also assume an $N$-subcarrier OFDM modulation scheme and assume that each subcarrier experiences flat fading. This models a typical cellular downlink transmission scenario. With the assumption that both transmit and receiver beamforming are performed, the demodulated signal for user $k$ on subcarrier $n$ is

$$
\hat{\mathbf{d}}_{k, n}=\mathbf{D}_{k, n}\left(\mathbf{H}_{k, n} \sum_{l=1}^{K} \mathbf{M}_{l, n} \mathbf{d}_{l, n}+\mathbf{n}_{k, n}\right)
$$

where $\mathbf{d}_{k, n}$ is the data vector for user $k$ on subcarrier $n, \mathbf{H}_{k, n}$ is the channel matrix, $\mathbf{M}_{k, n}$ is the transmit beamformer matrix, $\mathbf{D}_{k, n}$ is the receive beamformer matrix, and $\mathbf{n}_{k, n}$ is a complex white Gaussian noise vector.

The general MIMO-OFDMA resource allocation problem consists of determining which subcarriers are assigned to each user (in the MIMO case, several users will share each subcarrier), how much power is transmitted to each user on each subcarrier, and what transmit beamformers will be used. Solving this general problem in an optimal way is often intractable, except for very simple scenarios. In this paper, to simplify the solution, we use the block diagonalization (BD) method [11] to calculate the transmit beamformers, a technique which cancels out the inter-user interference at the transmit side. The resulting rate for user $k$ under BD in a MIMO- 
OFDMA setting will be

$$
R_{k}=\sum_{n=1}^{N} \log _{2}\left|\mathbf{I}+\frac{1}{N_{0}} \boldsymbol{\Sigma}_{k, n}^{2} \boldsymbol{\Lambda}_{k, n}\right|,
$$

where $N_{0}$ is the noise power, $\boldsymbol{\Sigma}_{k, n}^{2}$ is the diagonal matrix containing the singular values of user $k$ 's channel on subcarrier $n$, and $\boldsymbol{\Lambda}_{k, n}$ is the diagonal matrix with the power loading factors.

Even with the simplifications inherited from using block diagonalization, directly constructing a resource allocation bargaining problem from (2) leads to a nonlinear integer programming problem, which is difficult to solve. Hence we apply some relaxations to the original model and turn it into a convex programming problem. Note that, with $K$ users, there are a total of $2^{K}$ different user combinations that can be assigned to a given subcarrier $n$. Some of these combinations will not be feasible for $\mathrm{BD}$, since the sum of the number of receive antennas for users on a given subcarrier cannot exceed $n_{T}$. Suppose that after eliminating these infeasible cases, we are left with $I$ possible user combinations on any given subcarrier, and let $\left\{\varphi_{i, n}: n=1, \ldots, N\right.$ and $\left.i=1, \ldots, I\right\}$ denote the set of all possible user combinations over all subcarriers. Furthermore, let $0 \leq \omega_{n, i} \leq 1$ represent the fraction of the time that user combination $\varphi_{i, n}$ is used on subcarrier $n$. Similar approaches for modeling subcarrier allocations have been adopted in [12], [13]. Under these assumptions, the rate for user $k$ can now be expressed as

$$
\begin{aligned}
R_{k} & =\sum_{n=1}^{N} \sum_{i=1}^{I} \omega_{n, i} \log _{2}\left|\mathbf{I}+\frac{1}{\omega_{n, i} N_{0}} \boldsymbol{\Sigma}_{k, n, i}^{2} \boldsymbol{\Lambda}_{k, n, i}\right| \\
& =\sum_{n=1}^{N} \sum_{i=1}^{I} \omega_{n, i} \sum_{t=1}^{n_{R}^{(k)}} \log _{2}\left(1+\frac{\sigma_{k, n, i, t}^{2} \lambda_{k, n, i, t}}{\omega_{n, i} N_{0}}\right) .
\end{aligned}
$$

The above rate region can be shown to be convex.

\section{KALAI-SMORODINSKY BARGAINING SOLUTION}

We begin by explaining the fundamentals of bargaining in game theory and the Kalai-Smorodinsky approach [10], and then show how this approach can be applied to the MIMOOFDMA resource allocation problem.

A bargaining problem is defined as a pair $(U=$ $\left.\left(u_{1}, \ldots, u_{K}\right), \mathbf{d}\right)$, where $U \in \mathbb{R}^{K}$ is the set of feasible payoffs and $\mathbf{d} \in U$ is the status-quo or disagreement point. With $\mathcal{B}$ denoting the set of all possible bargaining problems, a bargaining solution is a function $f: \mathcal{B} \rightarrow U$. KSBS is the solution which satisfies four axioms: Pareto optimality, symmetry, invariance to affine transformations and individual monotonicity [10]. If $U$ is convex and compact, the KSBS must satisfy $\frac{u_{1}}{u_{1}^{\text {max }}}=\cdots=\frac{u_{K}}{u_{K}^{\text {max }}}$, where $\left\{u_{k}^{\max }\right\}_{k=1}^{K}$, called the "utopia" point, denotes the maximal achievable payoffs. For our problem, achieving $u_{k}^{\max }$ corresponds to allowing user $k$ to occupy all resources, and thus it is easy to determine.

We propose two different algorithms to find the KSBS for the MIMO-OFDMA problem. The first one is a bisection- search based method, while the second finds the solution by solving a sequence of related convex optimization problems.

\section{A. Bisection Search Algorithm}

From [10] we know the KSBS is the intersection point of the rate region boundary and the line segment from the origin to the utopia point. This fact was exploited by [14], in which a bisection search method was employed to find the solution. We adopt a similar approach here. For each search step, we check the feasibility of the bisection point. If the point passes the feasibility test, we move along the line and bisect the remaining segment. If the feasibility test fails we go back. This process repeats until the algorithm converges.

By introducing an artificial variable $s$, the feasibility test can be formulated as a standard convex optimization problem:

minimize $s$

$$
\begin{array}{ll}
\text { s.t. } & r-\frac{R_{k}}{R_{k}^{\text {max }}} \leq s, \forall k \\
& \omega_{n, i} \geq 0, \lambda_{k, n, i, t} \geq 0, \forall k, \forall n, \forall i, \forall t \\
& \sum_{i=1}^{I} \omega_{n, i}=1, \forall n \\
& \sum_{n=1}^{N} \sum_{i=1}^{I} \sum_{k \in \varphi_{i, n}} \sum_{t=1}^{n_{R}^{(k)}} \lambda_{k, n, i, t}=P
\end{array}
$$

where (8) is the service time constraint and (9) is the power constraint. Since it is a convex problem, a conventional algorithm [15] can be used to solve the feasibility test. If the optimal $s$ is not greater than 0 , the resource allocation vectors $\boldsymbol{\omega}$ and $\boldsymbol{\lambda}$ are feasible. Otherwise we claim that the feasibility test fails. When the algorithm terminates, we obtain the optimum rate ratio and the corresponding resource allocation. The bisection search algorithm is summarized in Algorithm 1.

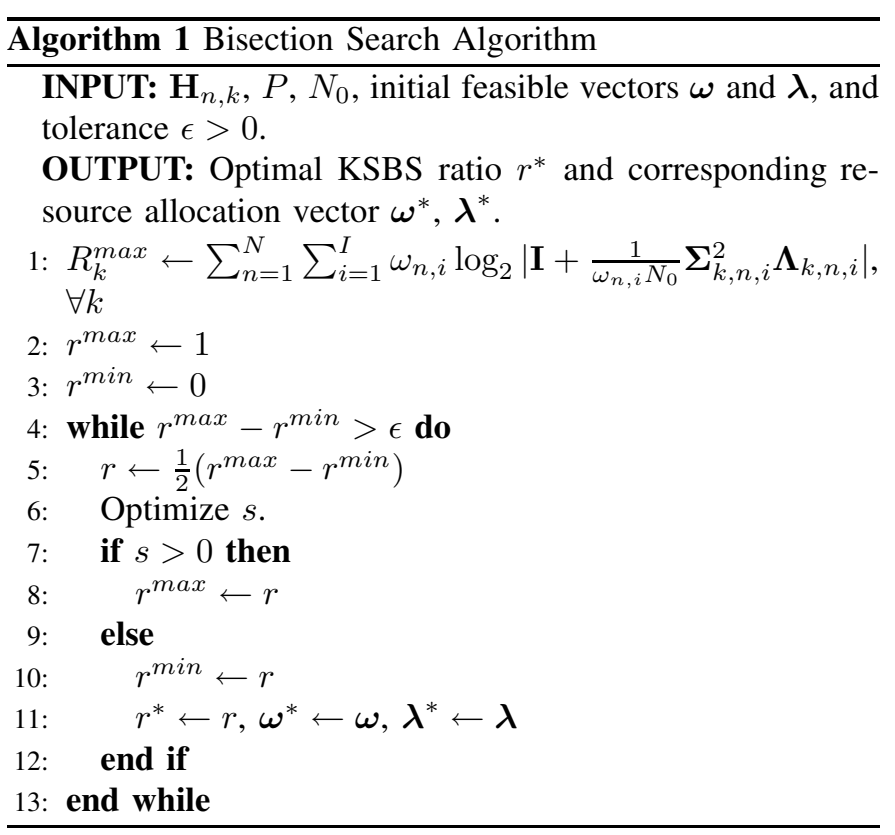




\section{B. Preference Function Based Algorithm}

In [16], the concept of preference functions was introduced. The author showed that different bargaining solutions can be generalized into a unified form by changing the weighting factor $\beta$ of the preference function. Different $\beta$ values correspond to different bargaining solutions. For example $\beta=0$ corresponds to the well-known Nash Bargaining solution and $\beta=1$ corresponds to the KSBS. For our resource allocation problem, the optimization problem can be formulated in terms of preference functions as

$$
\begin{aligned}
\operatorname{minimize} & -\log \prod_{k=1}^{K}\left[\frac{R_{k}}{R_{k}^{\text {max }}}+\frac{\beta}{K-1} \sum_{s=1, s \neq k}^{K}\left(1-\frac{R_{s}}{R_{s}^{\text {max }}}\right)\right] \\
\text { s.t. } & \omega_{n, i} \geq 0, \forall n, \forall i \\
& \lambda_{k, n, i, t} \geq 0, \forall k, \forall n, \forall i, \forall t \\
& \sum_{i=1}^{I} \omega_{n, i}=1, \forall n \\
& \sum_{n=1}^{N} \sum_{i=1}^{I} \sum_{k \in \varphi_{i, n}} \sum_{t=1}^{n_{R}^{(k)}} \lambda_{k, n, i, t}=P
\end{aligned}
$$

For any $\beta \neq 1$, this problem is strictly convex and we can directly solve it to obtain a unique solution. Unfortunately this is not possible when $\beta=1$, since in this case the solution is not unique and there is no easy way to tell if the optimum is the KSBS. However we can show the optimum for different $\beta$ values actually converges to the KSBS as $\beta \rightarrow 1$. Thus we consider solving the problem in an asymptotic manner.

Define

$$
p\left(\left\{R_{k}\right\}_{k=1}^{K}, \beta\right)=\prod_{k=1}^{K}\left[\frac{R_{k}}{R_{k}^{\text {max }}}+\frac{\beta}{K-1} \sum_{s=1, s \neq k}^{K}\left(1-\frac{R_{s}}{R_{s}^{\max }}\right)\right]
$$

and $p^{*}(\beta)=p\left(\left\{R_{k}^{*}(\beta)\right\}_{k=1}^{K}, \beta\right)$, where $\left\{R_{k}^{*}(\beta)\right\}_{k=1}^{K}$ is optimal for a given $\beta$. Due to the monotonicity of the $\log$ function, minimizing (10) is equivalent to maximizing $p\left(\left\{R_{k}\right\}_{k=1}^{K}, \beta\right)$.

Lemma 1: As $\beta \rightarrow 1, p^{*}(\beta)$ goes to 1 .

Proof: For an arbitrary $\beta$ and a feasible point $\left\{R_{k}\right\}_{k=1}^{K}$, we have

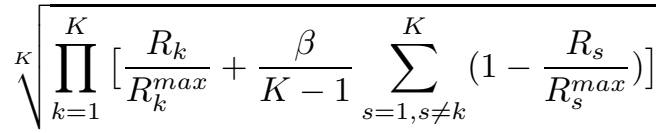

$$
\begin{aligned}
& \leq \frac{1}{K} \sum_{k=1}^{K}\left[\frac{R_{k}}{R_{k}^{\text {max }}}+\frac{\beta}{K-1} \sum_{s=1, s \neq k}^{K}\left(1-\frac{R_{s}}{R_{s}^{\text {max }}}\right)\right] \\
& =\beta+\frac{1}{K}(1-\beta) \sum_{k=1}^{K} \frac{R_{k}}{R_{k}^{\text {max }}} \leq 1,
\end{aligned}
$$

where (16) follows from the inequality of arithmetic and geometric means. Therefore $p\left(\left\{R_{k}\right\}_{k=1}^{K}, \beta\right) \leq 1$ holds for all feasible $\left\{R_{k}\right\}_{k=1}^{K}$.

$$
\text { If } \begin{aligned}
\frac{R_{1}}{R_{1}^{\max }}= & \frac{R_{2}}{R_{2}^{\max }}=\cdots=\frac{R_{K}}{R_{K}^{\max }}=r, \text { we have } \\
& p\left(\left\{R_{k}\right\}_{k=1}^{K}, \beta\right)=[r+\beta(1-r)]^{K}
\end{aligned}
$$

Since $\left\{R_{k}^{*}(\beta)\right\}_{k=1}^{K}$ achieves the maximum of $p\left(\left\{R_{k}\right\}_{k=1}^{K}, \beta\right)$, we know that

$$
[r+\beta(1-r)]^{K} \leq p^{*}(\beta) \leq 1 .
$$

The quantity $[r+\beta(1-r)]^{K}$ goes to 1 continuously as $\beta \rightarrow 1$, which means $p^{*}(\beta)$ also goes to 1 as $\beta \rightarrow 1$. The point sequence $\left\{R_{k}^{*}(\beta)\right\}_{k=1}^{K}$ converges since it is a continuous function of $\beta$.

Lemma 2: For any $\beta \in[0,1)$, the optimal solution $\left\{R_{k}^{*}(\beta)\right\}_{k=1}^{K}$ is a point on the boundary of the rate region.

Proof: We prove it by contradiction. If the optimum point does not lie on the boundary of the rate region, we choose $\Delta \in(0,1]$, for which $\left\{R_{k}^{*}(\beta)+\Delta \times R_{k}^{\max }\right\}_{k=1}^{K}$ is still feasible. Since the optimum point is not on the boundary, such $\Delta$ exists. Then we have

$$
\begin{aligned}
& \prod_{k=1}^{K}\left[\frac{R_{k}^{*}}{R_{k}^{\text {max }}}+\Delta+\frac{\beta}{K-1} \sum_{s=1, s \neq k}^{K}\left(1-\frac{R_{s}^{*}}{R_{s}^{\text {max }}}-\Delta\right)\right] \\
= & \prod_{k=1}^{K}\left[\frac{R_{k}^{*}}{R_{k}^{\text {max }}}+\frac{\beta}{K-1} \sum_{s=1, s \neq k}^{K}\left(1-\frac{R_{s}^{*}}{R_{s}^{\text {max }}}\right)+\Delta-\beta \Delta\right] \\
> & \prod_{k=1}^{K}\left[\frac{R_{k}^{*}}{R_{k}^{\text {max }}}+\frac{\beta}{K-1} \sum_{s=1, s \neq k}^{K}\left(1-\frac{R_{s}^{*}}{R_{s}^{\text {max }}}\right)\right]=p^{*}(\beta)
\end{aligned}
$$

which means $\left\{R_{k}^{*}(\beta)+\Delta \times R_{k}^{\max }\right\}_{k=1}^{K}$ outperforms $\left\{R_{k}^{*}(\beta)\right\}_{k=1}^{K}$, which contradicts the original assumption.

Lemma 3: The point to which $\left\{R_{k}^{*}(\beta)\right\}_{k=1}^{K}$ converges is on the boundary of the rate region.

Proof: To prove the limit point $\mathbf{R}^{*}$ is on the boundary, we need to show that in the rate region there exist points arbitrarily close to $\mathbf{R}^{*}$, and out of the rate region there also exist points arbitrarily close to $\mathbf{R}^{*}$ [15].

Let $\varepsilon=\varepsilon_{1}+\varepsilon_{2}$ be an arbitrary positive number. Since $\mathbf{R}^{*}$ is the limit point, we can always find a $\beta$ such that $\| \mathbf{R}^{*}(\beta)-$ $\mathbf{R}^{*} \|<\varepsilon_{1}$. This proves that there are arbitrarily close points in the rate region. Since $\mathbf{R}^{*}(\beta)$ is on the boundary, we can find $\mathbf{R}_{o}$ outside the rate region such that $\left\|\mathbf{R}^{*}(\beta)-\mathbf{R}_{o}\right\|<\varepsilon_{2}$. Therefore $\left\|\mathbf{R}^{*}-\mathbf{R}_{o}\right\| \leq\left\|\mathbf{R}_{o}-\mathbf{R}^{*}(\beta)\right\|+\left\|\mathbf{R}^{*}(\beta)-\mathbf{R}^{*}\right\|<$ $\varepsilon_{1}+\varepsilon_{2}=\varepsilon$. This proves that there are arbitrarily close points not in the rate region.

Theorem 1: $\left\{R_{k}^{*}(\beta)\right\}_{k=1}^{K}$ converges to the KSBS.

Proof: According to Lemma $1, \mathbf{R}^{*}$ is the solution to (10) for $\beta=1$. Since it is on the boundary of the rate region, we know that $\left\{R_{k}^{*}(\beta)\right\}_{k=1}^{K}$ converges to the KSBS.

This property can be exploited as summarized in Algorithm 2: First we choose an arbitrary $\beta \in[0,1)$ and check whether it is close enough to the actual KSBS. If not, we increase $\beta$ and repeat the optimization process. If it is, we can safely claim the solution is good enough and may be used as 


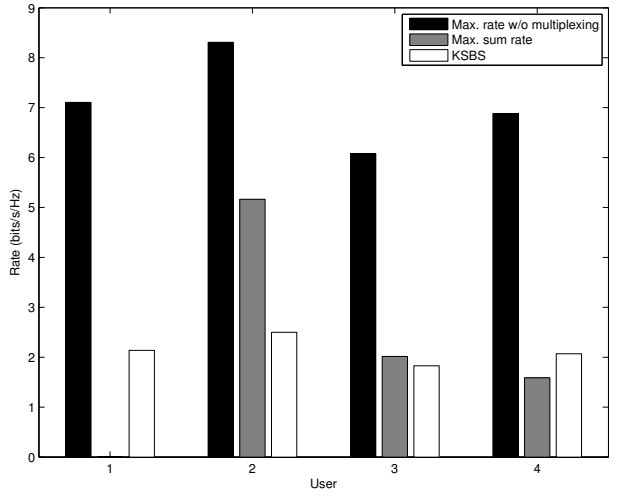

Fig. 1. Rate per user for sum rate and KS solutions

the real KSBS. Comparing this algorithm to Algorithm 1, the first approach is a search along the line from the origin to the utopia point while the second is a search along the boundary of the rate region.

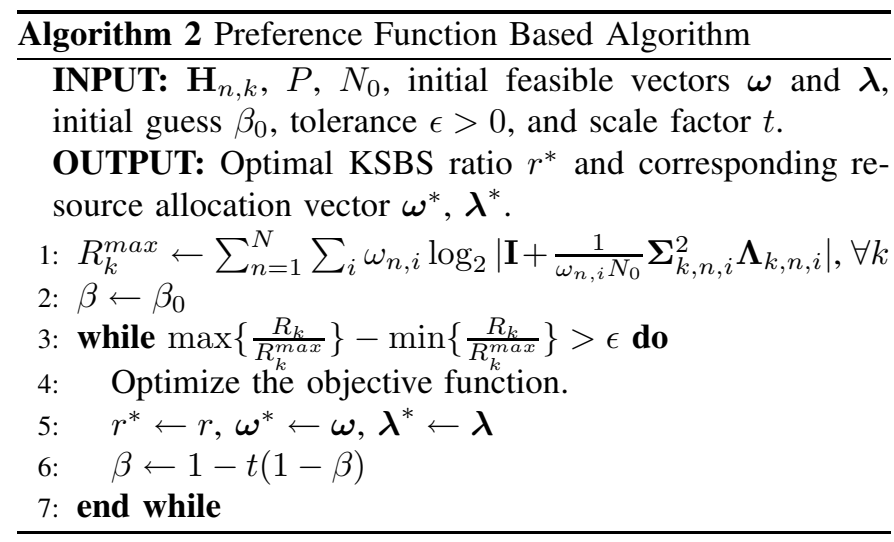

\section{Numerical Results AND CONCLUSION}

In Fig. 1 we compare the resource allocation outcome of the KSBS with the scheduling algorithm that maximizes the sum rate for a simple case where $K=4, N=3, n_{T}=4$ and $n_{R}=2$. The theoretical maximum rate $R_{k}^{\max }$ for each user is calculated and is also depicted in the figure. It can be seen that the sum rate achieved by KSBS is only slightly lower than that for the max sum rate algorithm, but there is a considerable improvement in fairness, especially for User 1.

To have a more complete view of the effectiveness of the KSBS, Fig. 2 illustrates the empirical cumulative distribution function (CDF) for 250 random channel realizations with $K=$ $2, N=8, n_{T}=2, n_{R}=1$ and equal path loss for every user. The CDF of the max sum rate algorithm is also given. The CDF curves clearly show that the KSBS provides a more fair solution and can achieve a good tradeoff between sum rate and fairness.

\section{CONCLUSION}

By adopting certain relaxations, we manage to convert the bargaining theory based MIMO-OFDMA resource allocation

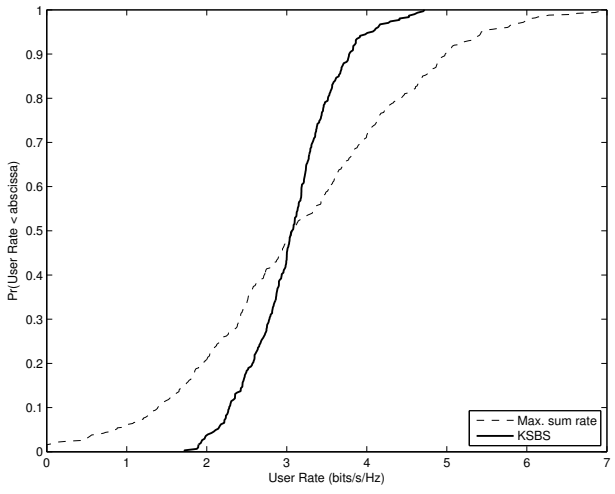

Fig. 2. Empirical CDF of users' rates for sum rate and KS solutions

problem into a convex programming problem. Two algorithms are proposed to find the KSBS and the convergence of the preference function based algorithm is also shown. The simulation results demonstrate that the KSBS can make a good tradeoff between equity and efficiency.

\section{REFERENCES}

[1] A. Goldsmith, S. Jafar, N. Jindal, and S. Vishwanath, "Capacity limits of mimo channels," Selected Areas in Communications, IEEE Journal on, vol. 21, no. 5, pp. 684-702, June 2003.

[2] H. Weingarten, Y. Steinberg, and S. Shamai, "The capacity region of the gaussian mimo broadcast channel," in Information Theory, 2004. ISIT 2004. Proceedings. International Symposium on, June-2 July 2004.

[3] M. Costa, "Writing on dirty paper (corresp.)," Information Theory, IEEE Transactions on, vol. 29, no. 3, pp. 439-441, May 1983.

[4] A. Tenenbaum and R. Adve, "Improved sum-rate optimization in the multiuser mimo downlink," in Information Sciences and Systems, 2008. CISS 2008. 42nd Annual Conference on, March 2008, pp. 984-989.

[5] P. Tejera, W. Utschick, G. Bauch, and J. Nossek, "Subchannel allocation in multiuser multiple-input-multiple-output systems," Information Theory, IEEE Transactions on, vol. 52, no. 10, Oct. 2006.

[6] S. Shi, M. Schubert, and H. Boche, "Rate optimization for multiuser mimo systems with linear processing," Signal Processing, IEEE Transactions on, vol. 56, no. 8, pp. 4020-4030, Aug. 2008.

[7] Y. J. Zhang and K. Letaief, "An efficient resource-allocation scheme for spatial multiuser access in mimo/ofdm systems," Communications, IEEE Transactions on, vol. 53, no. 1, pp. 107-116, Jan. 2005.

[8] E. Jorswieck and E. Larsson, "The miso interference channel from a game-theoretic perspective: A combination of selfishness and altruism achieves pareto optimality," in Acoustics, Speech and Signal Processing, 2008. IEEE International Conference on, 31 2008-April 42008.

[9] Z. Han, Z. Ji, and K. Liu, "Fair multiuser channel allocation for ofdma networks using nash bargaining solutions and coalitions," Communications, IEEE Transactions on, vol. 53, no. 8, pp. 1366-1376, Aug. 2005.

[10] E. Kalai and M. Smorodinsky, "Other solutions to nash's bargaining problem," Econometrica, vol. 43, no. 3, pp. 513-518, 1975.

[11] Q. Spencer, A. Swindlehurst, and M. Haardt, "Zero-forcing methods for downlink spatial multiplexing in multiuser mimo channels," Signal Processing, IEEE Transactions on, vol. 52, no. 2, Feb. 2004.

[12] P. Chan and R. Cheng, "Capacity maximization for zero-forcing mimoofdma downlink systems with multiuser diversity," Wireless Communications, IEEE Transactions on, vol. 6, no. 5, pp. 1880-1889, May 2007.

[13] W. Rhee and J. Cioffi, "Increase in capacity of multiuser ofdm system using dynamic subchannel allocation," in Vehicular Technology Conference Proceedings, 2000. VTC 2000-Spring Tokyo. 2000 IEEE 51st, vol. 2, 2000, pp. 1085-1089 vol.2.

[14] M. Nokleby and A. Swindlehurst, "Bargaining and the miso interference channel," EURASIP Journal on Advances in Signal Processing, 2009.

[15] S. Boyd and L. Vandenberghe, Convex Optimization. Cambridge University Press, 2004.

[16] X. Cao, "Preference functions and bargaining solutions," in Decision and Control, 1982 21st IEEE Conference on, vol. 21, Dec. 1982. 